The results of all this labour are shown by the Aiagram ( $\mathrm{p}$ 258 ), and also by the following table.

\begin{tabular}{cclll} 
Temp. & & \multicolumn{1}{c}{ Old. } & & \multicolumn{1}{c}{ Corrected. } \\
IO $^{c}$ & $\ldots$ & $4200 \times 10^{4}$ & $\ldots$ & $4197 \times 10^{1}$ \\
I5 & $\ldots$ & 4189 & $\ldots$ & 4189 \\
20 & $\ldots$ & 4179 & $\ldots$ & 4183 \\
25 & $\ldots$ & 4173 & $\ldots$ & 4177 \\
30 & $\ldots$ & 4171 & $\ldots$ & 4173 \\
35 & $\ldots$ & 4173 & $\ldots$ & $4 I 74$
\end{tabular}

Griffiths.

... $4199^{\circ} 7 \times 10^{4}$

... $4 \mathrm{II}^{\circ} 2$

... $4187^{\circ} 4$

Prof. Schuster and Mr. Gannon did not extend their determinations over any appreciable temperature range, and it is impossible, therefore, to apply the "rate of change" comparison to their results. Their thermometry, however, was directly based on the International Standard, hence it is probable that we now have three separate determinations of the "mechanical equivalent" in which the temperature scale is identical.

It appears to me that the data now at our disposal justify the following conclusions :-

(a) That the rate of change in the capacity for heat of water from $10^{\circ}$ to $25^{\circ} \mathrm{C}$. may be considered as known with sufficient accuracy for present purposes.

(b) That the persistent difference in the capacity for heat of water, when determined by mechanical and electrical methods, indicates a possibility that there is some error in one of the electrical units. ${ }^{1}$

One further matter deserves attention, concerning which there has hitherto existed considerable uncertainty, viz. the value of

the ratio

$$
\text { "mean thermal unit" }
$$

The value of this ratio is of great importance, as in the absence of exact information on this point we are unable to utilise the results of many notable experiments, suci as those performed with Bunsen's calorimeter.

Until recently Regnault's value ( $\mathrm{I} \cdot 005$ in terms of the thermal unit $0^{\circ}$ to $\mathrm{r}^{\circ}$ ) has been universally adopted.

Some experiments performed by me in 1894 , on the latent heat of evaporation of water, led to the cunclusion that "the value of the 'mean thermal unit' is practically identical with that at $15^{\circ}$ C." (Trans. Roy. Soc., 1895, p. 320).

On the publication of this statement, Dr. Joly performed some experiments, from which we cbtain the value 9962 in terms of the thermal unit at $15^{\circ}$ C. (Phil. Mag., November 1895, p. 440), assuming Rowland's uncorrected values from $0^{\circ}$ to $15^{\circ}$. $^{2}$

In the Report of the Electrical Standards Committee, I 896 , Mr. Shaw gives the results of a recalculation of Regnault's numbers, in which, assuming Rowland's uncorrected values, he obtains 1.0016 in terms of the thermal unit at $10^{\circ}$.

The enumeration of these facts indicates the extent of our uncertainty, and here again recent investigations lead us on to firmer ground.

In the Bakerian lecture delivered by Prof. Osborne Reynolds, on May 20, 1897 , he communicated the results of an investigation by Mr. Moorby and himself into the value of the " mean thermal unit." Their results are of peculiar importance because they are practically independent of temperature measurements, and also on account of the large scale on which they were conducted. Their conclusion is " 776.94 mechanical units at Manchester." I take this as equal to $777^{\circ} \mathrm{O} 7$ at Greenwich $=4184 \times 10^{4}$ ergs. This is about equal to Rowland's corrected value at $19^{\circ}$, or, expressing it in terms of Rowland's corrected value at $15^{\circ}$, we get 9988, which is sufficiently near to unity to justify my prediction as to the practical equality of the two units.

I trust that the above short summary will suffice to show that great advances have recently been made, and I venture to express a hope that the importance of extreme accuracy with regard to thermal measurements may, in the future, be more generally recognised than, I believe, has been the case in the past.

E. H. Griffiths.

1 For example, an error of $\mathrm{x}$ in 1000 in the electro-chemical-equivalent of silver would account for nearly the whole of our present discrepancies.

2 I find that if we take Rowland s corrected values, this number approximates to 9975 .

NO. I 446 , VOL. 56$]$

\section{THE ACTION OF LIGHT ON DIASTASE.}

$T H E$ influence of the different rays of the solar spectrum upon the various phenomena of vegetable life has been shown by niany observers to be not at all uniform. Speaking broadly, the rays lying to the left of the green, often collectively termed those of the red end, have been found to be most actively concerned with the metabolic processes. They are the ones on which generally the working of the chlorophyll apparatus depends, and in their absence no construction of carbohydrates from the carbonic anhydride of the air takes place. The rays beyond the green to the right, including also the ultra-violet ones, have, on the other hand, been shown to play but a small part in such constructive processes, but to be those on which the phenomena of heliotropism and other interferences with growth depend. They are broadly associated, therefore, with the physical rather than the chemical processes.

In recent years the influence of the blue, violet and ultraviolet rays has been found to be deleterious to vegetable protoplasm, exposure to them destroying many micro-organisms. They have further been shown to share, though to a small extent only, the power of assisting the chlorophyll apparatus. Some time ago a research of considerable importance was conducted by Messrs. Brown and Morris, from which it appeared that the amount of diastase obtainable from foliage leaves varies considerably at different periods of the day, being greater after darkness, and diminishing after exposure to light. An investigation of the action of the different rays of the spectrum on diastase has recently been carried out by Prof. Reynolds Green, which shows that its separate regions possess radically different powers, and that while some rays are beneficial and aid in the secretion of the enzyme, others are as distinctly deleterious and, indeed, affect the diastase in the same way as those of the blue end do the micro-organisms already spoken of.

By the use of appropriate screens the spectrum was divided into seven bands, the infra-red, the red, including the rays of wave-length $720 \mu \mu$ to $640 \mu \mu$; the orange, ranging from $640 \mu \mu$ to $585 \mu \mu$; the green, from $585 \mu \mu$ to $500 \mu \mu$; the blue, from $500 \mu \mu$ to $430 \mu \mu$; the violet, including the visible rays beyond wave-length $430 \mu \mu$; and the ultra-violet.

Solutions of diastase, prepared from malt extract, and from the leaves of Phaseolus vulgraris, as well as from human saliva, were exposed to strong illumination, either that of the sun, or of a strong naked electric arc-lamp, for several hours, and after such exposure their hydrolytic power was tested side by side with that of colıcrol solutions that had not been illuminated. By the use of the screens already mentioned, the effects of these several regions of the spectrum were ascertained.

Of the infra-red, red,orange and blue regions the rays were found to have a distinctly beneficial effect upon the manufacture of the enzyme. The effect was, however, much the greatest in the red region, these rays, when allowed to act for ten to twelve hours, increasing the amount of diastase by one-half. A second maximum was obtained in the blue, while the rays of the other regions were intermediate in power.

The violet and ultra-violet rays were ascertained to exert a destructive action on the enzyme, an exposure of ten to twelve hours to the latter especially, often resulting in the destruction of 60 per cent. of the diastase. The change set up in the solution was found to be a continuous and progressive one, the extract becoming weaker after removal from the action of the light until the diastasic power entirely disappeared.

This effect was found to follow also a strong illumination of the living leaf. Leaves of Phaseolus were exposed to sunlight and the electric arc respectively, half of each being carefully shaded by a cover of blackened paper. After the illumination, weighed quantities of the shaded and unshaded sides were made to act under the same conditions upon a solution of starch, when the hydrolytic power of the unscreened portions was found to have been very materially injured.

The difference in the action of the various rays suggests a modification of the view that the chemical action of light in vegetable metabolism is particularly a property of the red end of the spectrum. This is borne out further by some experiments recently published by Laurent, who has found that the blue and violet rays are especially active in the construction of nitrogen-containing compounds in the plant. All the rays appear to be able to bring about chemical effects, the differences depending upon the materials taking part in the reactions, rather than upon any radical differences in the nature of the light. 